\title{
Three-Dimensional Surface Profile Measurement of Microlenses Using the Shack-Hartmann Wavefront Sensor
}

\author{
Chenhui Li, Gunnsteinn Hall, Difeng Zhu, Heng Li, Kevin W. Eliceiri, and Hongrui Jiang, Senior Member, IEEE
}

\begin{abstract}
We present a 3-D surface profiling method for microlenses that utilizes a Shack-Hartmann wavefront sensor. This method applies to both solid microlenses and liquid-liquid interfaces in liquid microlenses. The wavefront at the aperture stop of a microlens is measured by a Shack-Hartmann wavefront sensor and is then used to calculate the 3-D surface profile of the microlens. Three types of microlenses-a photoresist microlens, a hydrogel-driven tunable liquid lens, and an electrowettingdriven tunable liquid lens-were fabricated and measured. The variable-focus liquid lenses were tested within a wide focal length range. The obtained surface profiles were fitted to spherical and conical surface models to study their geometrical properties. The photoresist microlens was found to be approximately spherical. For the hydrogel-driven microlens, the profile was smooth and nearly spherical at the center but became steep and linear at the aperture edges. The electrowetting-driven liquid lens was also fitted better with the conical model, and its conic constant was determined. The obtained surface profiles were used to estimate the optical properties of microlenses in an optical analysis software package. The comparison between the simulation and experiment results indicated that the accuracy of the estimation is rough and the error could be due to the wavefront measurement and surface fitting approximation.

[2011-0321.R1]
\end{abstract}

Index Terms-Electrowetting (EW), hydrogel, liquid microlens, Shack-Hartmann sensor, three-dimensional (3-D) surface profile, variable focus.

Manuscript received October 31, 2011; revised January 9, 2012; accepted January 15, 2012. Date of publication February 17, 2012; date of current version May 28, 2012. This work was supported in part by the National Science Foundation (NSF) under Grant ECCS 0745000 and Grant EFRI 0937847, in part by the National Institutes of Health under Grant CA136590, and in part by the Wisconsin Institutes for Discovery. Subject Editor S. Merlo.

C. Li is with the Department of Electrical and Computer Engineering, University of Wisconsin, Madison, WI 53706 USA.

G. Hall is with the Department of Biomedical Engineering and also with the Laboratory for Optical and Computational Instrumentation, University of Wisconsin, Madison, WI 53706 USA.

D. Zhu was with the Department of Electrical and Computer Engineering, University of Wisconsin, Madison, WI 53706 USA. He is now with Micron Technology Inc., Boise, ID 83707 USA.

$\mathrm{H}$. Li is with the Micro-Nano Sensors and Actuators Laboratory, Department of Electrical and Computer Engineering, University of Wisconsin, Madison, WI 53706 USA.

K. W. Eliceiri is with the Laboratory for Optical and Computational Instrumentation, Eye Research Institute, and also with the Department of Biomedical Engineering, University of Wisconsin, Madison, WI 53706 USA.

$\mathrm{H}$. Jiang is with the Department of Electrical and Computer Engineering, the Department of Biomedical Engineering, Materials Science Program, and also with the Eye Research Institute, University of Wisconsin, Madison, WI 53706 USA (e-mail: hongrui@engr.wisc.edu).

Color versions of one or more of the figures in this paper are available online at http://ieeexplore.ieee.org.

Digital Object Identifier 10.1109/JMEMS.2012.2185821

\section{INTRODUCTION}

$\mathbf{T}$ HE TREND toward the miniaturization of optics and electro-optics has made the rapidly evolving microlenses technology important components in both commercial and research-based optical systems. Microlenses play a crucial role in many applications, such as portable cameras [1], [2], beam shaping for wavefront correction [3], [4], optical array interconnection [5], [6], microlens projection lithography [7], [8], and wavefront sensors [9]. Different types of microlenses have been developed. Some microlenses have fixed focus and are normally made of solid materials [10]-[12]. Benefitting from variable focus, high transmission, and compact structure, liquid microlenses have drawn extensive research attention and have been demonstrated by various mechanisms such as electrowetting (EW) [1], [2], [13], stimuli-responsive hydrogels [14]-[16], and pressure-driven techniques [17]-[21]. During the fabrication process, the surface shape of the microlenses is a key concern, because theoretically, it determines the optical properties of a microlens, assuming that the properties of lens material are uniform and stable across its body. For liquid microlenses, the geometrical surface is influenced by several factors such as the design of geometric structure [2], the control of surface wetting [22], the effect of gravity [23], and the surface smoothness. Thus, a good knowledge of the 3-D surface profile is critical for liquid microlens design and the optimization of the fabrication process. Surface profiles are also beneficial to gain a deeper understanding of the surface deformation of variable-focus microlenses [20]. Surface profiles of solid microlenses are normally measured by mechanical profilometers [10], [20] or white-light interferometers [10], [17], [24]. However, these approaches are not applicable to liquid-liquid interfaces. For some solid lenses made of soft materials, a thin film of aluminum or gold usually needs to be presputtered on the surface to enhance the reflection so that clear interferometric images can be obtained [24]. This measurement is essentially invasive. Conventional goniometers cannot perform 3-D measurements and require high optical transparency on the surrounding structure of the microlens. Hence, currently, there is great need for an accurate convenient method for 3-D surface profile measurement of microlenses.

To help address this need, we previously reported on preliminary results on the use of a Shack-Hartmann wavefront sensor to measure the 3-D surface profiles of the liquid-liquid interface in a liquid microlens [25]. Here, we present detailed, expanded results on a complete 3-D surface profiling method 
(a)

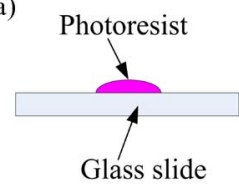

(b)

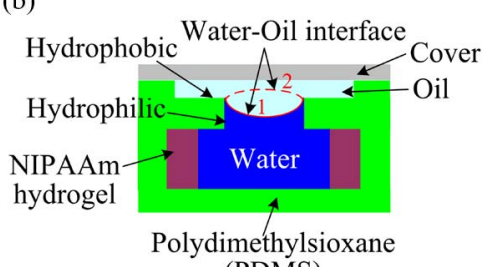

(PDMS)

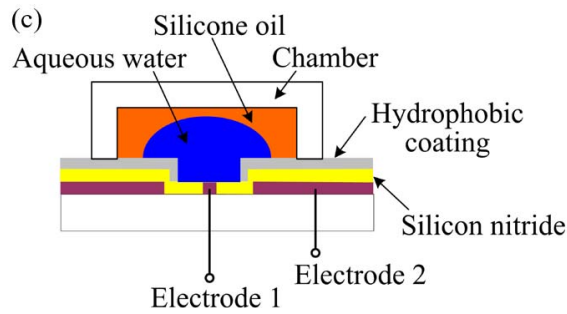

Fig. 1. Cross-sectional schematic of the three types of microlenses tested. (a) Photoresist microlens. (b) Hydrogel-driven liquid microlens. The water-oil interface is pinned at the hydrophilic-hydrophobic boundary. The NIPAAm hydrogel is thermoresponsive. Its focal length can be changed by temperature variation. It functions as a converging lens at status 1 and as a diverging lens at status 2. (c) Electrowetting-driven microlens. Its focal length is varied by the voltage applied on the electrodes.

using a Shack-Hartmann wavefront sensor. This approach is applicable to both solid and liquid microlenses, and the optical setup can easily be adjusted to measure microlenses in different dimensions. A physical model is presented to derive the surface profile from the wavefront profile. The following three types of microlenses were fabricated and tested: 1) a solid photoresist lens; 2) a hydrogel-driven liquid lens, and 3 ) an EW-driven liquid lens. Fig. 1 shows the cross-sectional schematic of each type of microlens. Their surface shapes are all primarily determined by surface tension, and they could be influenced by surface wettability and the effects of gravity to some degree. The measured surface profiles were fitted to spherical and conical surface models to quantitatively analyze the geometrical properties, and the fitted surface models can be used to roughly estimate the focal length and aberrations of the microlenses.

\section{PRINCIPLES}

Light propagation can be described with Maxwell's equations. The incident wavefront through a lens is delayed by an amount proportional to the optical path length (OPL) that it has traveled [26]. For a planoconvex/concave lens, if the lens material is uniform, the OPL through each path is determined by its surface profile and refractive index. Therefore, the surface profile of a microlens can be derived when the incident and refracted wavefronts are both known. Fig. 2 illustrates the wavefront refraction of a plane wave through a microlens and the principles of surface profile calculation of a microlens. The lens consists of two materials $\mathrm{M}_{1}$ and $\mathrm{M}_{2}$ with refractive indices $n_{1}$ and $n_{2}$, respectively. The $M_{1}$-to- $M_{2}$ interface functions as a lens, and it can be either a diverging or a converging lens, depending on its curvature and refractive index difference between $M_{1}$ and $M_{2}$ [1], [14]. This is a generalized physical model of commonly used liquid and solid microlenses. For liquid lenses, $M_{1}$ and $M_{2}$ are two immiscible liquids with

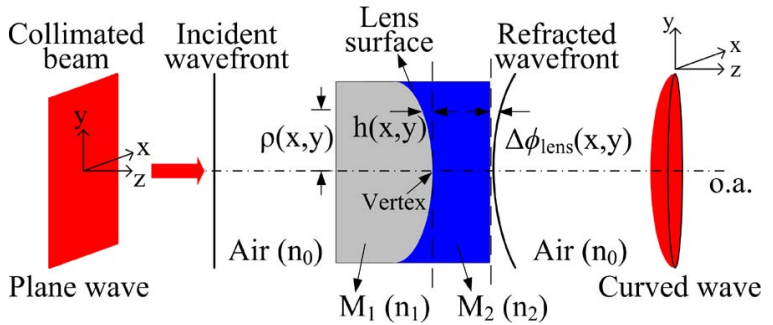

Fig. 2. Principle of 3-D surface profile measurement. For a solid microlens, $M_{1}$ is the lens material, and $M_{2}$ is air. For a liquid microlens, $M_{1}$ and $M_{2}$ are two immiscible liquids. The microlens is illuminated by a collimated beam, and it is tested in the transmission mode. The incident wavefront is a plane wave. The refracted wavefront $\left(\Delta \phi_{\text {lens }}(x, y)\right)$ is curved, and its shape is determined by the surface profile and refractive indices of $M_{1}$ and $M_{2}$. The surface profile of the microlens can be calculated from the refracted wavefront by (2).

different refractive indices. For a solid lens, $\mathrm{M}_{1}$ is a solid material, and $\mathrm{M}_{2}$ is air. At the aperture stop of a microlens, the optical path difference (OPD) between the origin and point $(x, y)$ in the plane is

$$
O P D(x, y)=\left(n_{1}-n_{2}\right) \times h(x, y)
$$

where $h(x, y)$ is the thickness difference of $\mathrm{M}_{2}$ between the horizontal path through the vertex and through $(x, y)$, and $n_{1}$ and $n_{2}$ are the refractive indices of $\mathrm{M}_{1}$ and $\mathrm{M}_{2}$, respectively. The 3-D surface profile of the lens, described by $h(x, y)$, can be calculated from the refracted wavefront $\Delta \phi_{\text {lens }}(x, y)$, and the equation that has been derived is [25]

$$
h(x, y)=\frac{O P D(x, y)}{n_{1}-n_{2}}=\frac{\Delta \phi_{\text {lens }}(x, y) \times \lambda / 2 \pi}{n_{1}-n_{2}}
$$

where $\lambda$ is the wavelength of the light source.

To analyze the surface properties of the microlenses, the measured surface profiles can be fitted to the following two geometric models: 1) a spherical surface model and 2) a conical surface model. As an approximation, it is normally assumed that microlenses are spherical lenses. Given that the vertex of a sphere is at $(x, y, z)=(0,0,0)$, a spherical surface profile is described by

$$
z=\frac{c\left(x^{2}+y^{2}\right)}{1+\sqrt{1-c^{2}\left(x^{2}+y^{2}\right)}}
$$

where $z$ is the height of the surface, and $c$ is the reciprocal of $R$, the radius of curvature ( $\mathrm{RoC})$.

In reality, due to fabrication factors, the nonuniformity of surface wetting, and the effects of gravity, the surface profile of a microlens often deviates from the spherical shape in the area away from the center. A conical surface model is often used to account for such surface variations, and it is described by

$$
z=\frac{c\left(x^{2}+y^{2}\right)}{1+\sqrt{1-c^{2}(1+k)\left(x^{2}+y^{2}\right)}}
$$

where $k$ is the conic constant and specifies spherical $(k=0)$, elliptical $(-1<k<0)$, parabolic $(k=-1)$, and hyperbolic $(k<-1)$ surfaces. 
For a spherical lens, the focal length $f$ is determined by $R$ and the refractive index difference between $\mathrm{M}_{1}$ and $\mathrm{M}_{2}$, as shown in

$$
f=\frac{R}{n_{1}-n_{2}} .
$$

For the liquid lenses tested, $n_{1}$ and $n_{2}$ are the refractive indices of silicone oil $\left(n_{1}=1.48\right)$ and water $\left(n_{2}=1.33\right)$. For the photoresist microlens tested, $n_{1}=1.65$ (refractive index of AZ4620), and $n_{2}=1$ (refractive index of air). For a microlens of a conical surface shape, (5) can also be used to calculate the focal length when the paraxial approximation is valid.

\section{Optical SETUP AND PRINCIPLES OF THE WAVEFRONT SENSOR}

\section{A. Optical Setup and Surface Profile Calculation}

The conceptual diagram of the optical setup for wavefront measurements is shown in Fig. 3(a) [25]. A collimated laser beam is first expanded by a beam expander and then illuminates the microlens sample, which is tested in the transmission mode [27]. The wavefront in the aperture stop of the microlens is transferred by relay lenses to the lenslet array in the Shack-Hartmann wavefront sensor. The microlens in Fig. 3(a) has a large optical power so that the beam that emerges from the microlens crosses its focal spot before reaching the first relay lens $\mathrm{L}_{1}$. The dynamic range of the wavefront sensor constrains the maximum optical power of a microlens that can be tested (a detailed discussion is shown in Section VII). Depending on the aperture size of the microlens, the relay lenses are designed to expand the refracted beam out of the microlens so that a significant portion of the charge-coupled device (CCD) sensor is filled. A higher filling ratio of the CCD sensor provides a higher sampling and a higher resolution of the wavefront. The magnification factor $M$ of the relay lenses is

$$
M=-\frac{f_{2}}{f_{1}}
$$

where $f_{1}$ and $f_{2}$ are the focal length of the two relay lenses, respectively, and the minus sign accounts for the image inversion. The aperture stop plane (LL') of the microlens and the lenslet array plane $\left(\mathrm{SS}^{\prime}\right)$ of the wavefront sensor are conjugate planes, which means that $\mathrm{LL}^{\prime}$ is at the front focal plane of relay lens $L_{1}$ and $S^{\prime}$ is at the back focal plane of relay lens $L_{2}$. Here, it is assumed that the lens structure is very thin so that the wavefront at LL', i.e., a flat substrate, is approximately the same as the wavefront at the curved lens surface $\left(\mathrm{CC}^{\prime}\right)$. For the microlenses tested in this paper, the thickness between the curved lens surface and the flat exit surface of the microlens is a few hundred micrometers. The relationship between the wavefront at $\operatorname{LL}^{\prime}\left(\Delta \phi_{\text {lens }}\right)$ and the wavefront measured by the wavefront sensor $\left(\Delta \phi_{\text {sensor }}\right)$ is described by

$$
\Delta \phi_{\text {lens }}(x, y)=\Delta \phi_{\text {sensor }}(M \times x, M \times y) .
$$

Therefore, the wavefront measured by the sensor needs to be rescaled to obtain the wavefront at the microlens. Combining
(2) and (7), the surface profile of the microlens is calculated from the measured wavefront by

$$
h(x, y)=\frac{\Delta \phi_{\text {sensor }}(M \times x, M \times y) \times \lambda / 2 \pi}{n_{1}-n_{2}} .
$$

Fig. 3(b) shows two pictures and the layout of the experimental setup. The light source was a helium-neon ( $\mathrm{HeNe})$ laser $(\lambda=594 \mathrm{~nm})$, and the beam was enlarged to around $2 \mathrm{~mm}$ in diameter by a beam expander. Relay lenses and a Shack-Hartmann wavefront sensor (WFS150C, Thorlabs Inc., Newton, NJ) were fixed on a horizontal optical rail. The microlens was placed on a vertical stage. Four mirrors were used to redirect the beam so that the collimated beam went straight through the microlens to be tested. An optical intensity filter (not drawn in the figure) was inserted after the beam expander when the light saturated the wavefront sensor.

\section{B. Shack-Hartmann Wavefront Sensor}

Shack-Hartmann wavefront sensors are commonly used for wavefront characterization and measuring lens aberrations [28]-[30]. The sensor consists of a microlens (lenslet) array and a photon detector for position sensing [31]. All the lenslets have the same focal length, and the photon detector is typically a CCD array. The incoming light is sampled by the lenslet array and then focused onto the CCD, creating a number of separated focal spots of light on the CCD. The position of the focal spot within each pixel of the CCD is used to calculate the local wavefront slope and then to reconstruct the whole wavefront. Fig. 3(c) illustrates the internal structure of the Shack-Hartmann wavefront sensor used in the experiment. The CCD is a $4.49 \mathrm{~mm} \times 4.49 \mathrm{~mm}$ square, and the resolution of the surface measurement is $150 \mu \mathrm{m} / M$, determined by the lenslet aperture and the magnification factor $M$ of the relay optics [defined in (6)]. According to the manufacturer specifications of the wavefront sensor, the accuracy of the wavefront measurement is $\lambda / 15$ or $39.6 \mathrm{~nm}$ for $\lambda=594 \mathrm{~nm}$.

Before each microlens was measured, the wavefront sensor was first calibrated to subtract the systematic aberration from the wavefront measurement. The calibration was made using the setup in Fig. 3(b) without the microlens to be tested, and the calibration using a plane wavefront was chosen in the built-in software of the Shack-Hartmann wavefront sensor.

\section{Verification of Principles and Optical Setup}

To test the reliability and accuracy of the physical model and the optical setup, a commercial plastic lens $(n=1.491$, AOI-0814, Align Optics Inc., Sunrise, FL) was first measured, and the obtained surface profile was compared with the lens profile reported by the manufacturer. This lens is a planoconvex spherical lens $(R=14.6 \mathrm{~mm})$. Relay lenses with a magnification factor of $M=2$ were used in this measurement. The surface profile obtained is fitted to a spherical surface defined by (3). The implied $R$ is $14.1 \mathrm{~mm}$, resulting in a relative error of $3.80 \%$. The root mean square (rms) of the difference between the manufacturer-reported profile and the experiment result is $1.4 \mu \mathrm{m}$. A commercial glass microlens $(n=1.516$, NT47-381, 


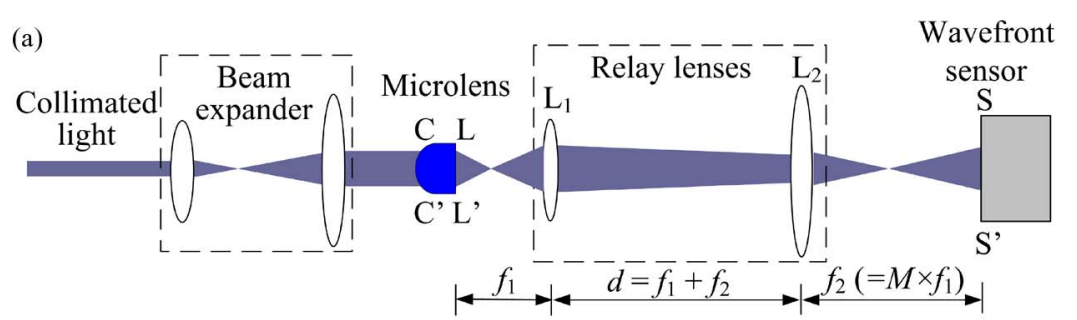

(b)

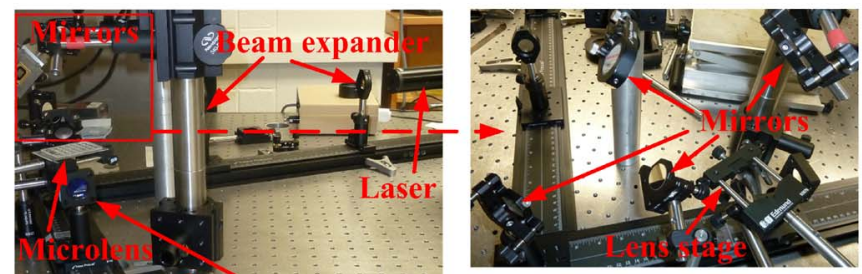

(c) Wavefront sensor

Lenslet Array CCD Sensor
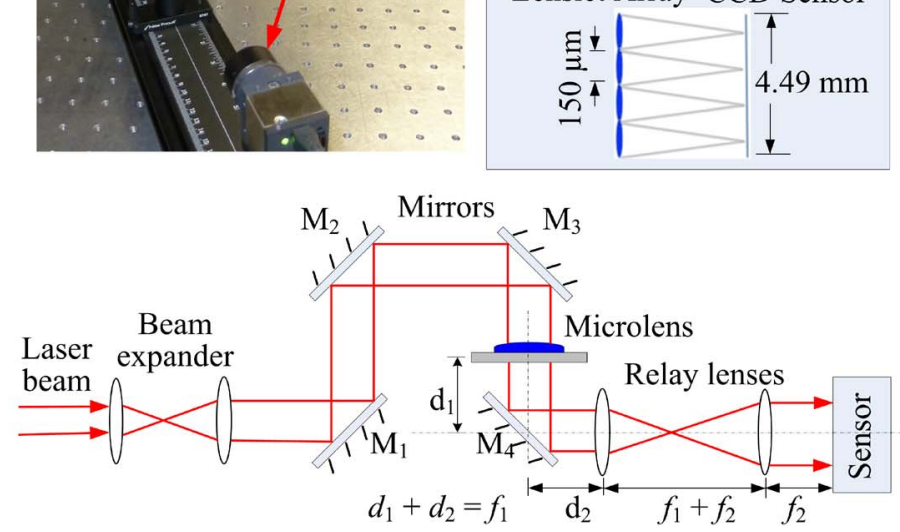

Fig. 3. (a) Diagram of the optical setup for wavefront measurement. A collimated beam is expanded by a beam expander, and then, it illuminates the microlens. The aperture stop plane of the microlens $\left(\mathrm{LL}^{\prime}\right)$ and the wavefront sensor plane $\left(\mathrm{SS}^{\prime}\right)$ are conjugate planes of the relay lenses. The image at $\mathrm{LL}^{\prime}$ is enlarged by a factor of $M$. (b) Pictures of the optical setup. The microlens to be tested was placed on a vertical stage. The relay lenses and the wavefront sensor were fixed on a horizontal optical rail. A few mirrors were used to redirect the light. (c) Internal structure of the wavefront sensor. The front of the sensor is a lenslet array followed by a CCD sensor $(4.49 \mathrm{~mm} \times 4.49 \mathrm{~mm})$. The aperture of each single lens in the lenslet array is $150 \mu \mathrm{m}$.

Edmund Optics, Barrington, NJ) of high surface quality was also tested, and the experiment result gave a $0.97 \%$ relative error of $R$ and a $0.105-\mu \mathrm{m}$ rms of the profile error, close to the theoretical accuracy limit $(77 \mathrm{~nm})$ of this measurement [26].

\section{FABRICATION OF MiCROLENSES}

The following three types of microlenses were fabricated: 1) a photoresist lens; 2) a liquid lens actuated by thermoresponsive hydrogel; and 3) an EW-driven liquid lens. The photoresist microlens is a fixed-focus converging lens. The hydrogel-driven variable-focus liquid lens can be actuated to either a converging or a diverging lens, depending on the local temperature [14]. The EW-driven lens is a variable-focus diverging lens, and its focal length changes with the voltage applied [1], [12].

\section{A. Photoresist Microlens}

The photoresist microlens was fabricated by a standard photolithography technique and thermal reflow process [10], [11]. The fabrication process is illustrated in Fig. 4(a)-(c). Thickfilm photoresist AZ4620 (AZ P4620, AZ Electronic Materials, Branchburg, NJ) was spin coated on a microscope slide at
$2000 \mathrm{r} / \mathrm{min}$ for $30 \mathrm{~s}$ and then exposed to ultraviolet (UV) light through a film mask (IMAGESETTER Inc., Madison, WI) at $20 \mathrm{mWcm}^{-2}$ for $20 \mathrm{~s}$. Next, the sample was developed in an AZ 400k developer for $180 \mathrm{~s}$ to form $9-\mu$ m-high cylindrical photoresist pads on the substrate. Then, they were heated on a hot plate at $160^{\circ} \mathrm{C}$ for $10 \mathrm{~min}$ to melt the photoresist pedestals, and finally, they were cooled down to form the photoresist microlenses. Fig. 4(d) shows a picture of the photoresist microlenses, and the focal length of each of these converging lenses was measured to be around $4.5 \mathrm{~mm}$.

\section{B. Liquid Microlens Actuated by a Thermoresponsive Hydrogel}

The fabrication of a hydrogel-driven variable-focus liquid microlens is based on liquid-phase photopolymerization and soft lithography [14]. The process detail was reported earlier [15]. The cross-sectional schematic of the microlens is shown in Fig. 1(b). At the lens aperture, a water-oil meniscus was pinned at the hydrophobic-hydrophilic boundary. The refractive index of silicone oil $\left(n_{1}=1.48\right)$ is larger than the refractive index of water $\left(n_{2}=1.33\right)$. Therefore, when the water-oil interface was bending downward [status 1 in Fig. 1(b)], the microlens 
(a) Spin coating

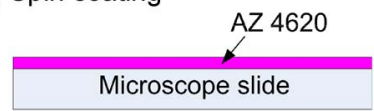

(b) Photolighthography

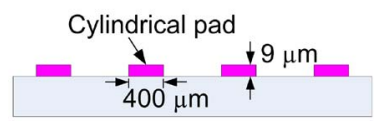

(c)

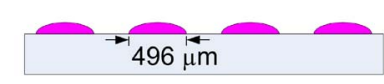

(d)

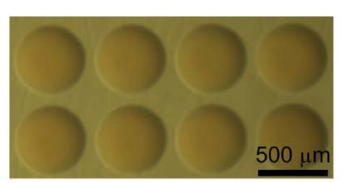

Fig. 4. Fabrication of the photoresist microlens. (a) Thick photoresist was spin coated on a microscope slide at $2000 \mathrm{r} / \mathrm{min}$ for $30 \mathrm{~s}$. (b) Photoresist film was photopatterned. After it was developed, 9- $\mu$ m-high cylindrical photoresist pads were left on the substrate. (c) Photoresist pads were heated at $160^{\circ} \mathrm{C}$ for $10 \mathrm{~min}$. During the process, the photoresist pads were melted and then reflowed to form a microlens when it was cooled down. The diameter of the microlens was around $496 \mu \mathrm{m}$. (d) Picture of the photoresist microlenses.

functioned as a converging lens, and when the interface was bulging up [status 2 in Fig. 1(b)], it was a diverging lens. The net volume of the $\mathrm{N}$-isopropylacrylamide (NIPAAm) hydrogel and the water in the cavity changes with temperature variations, thus varying the focal length.

\section{EW-Driven Liquid Microlens}

Fig. 5 illustrates the fabrication of an EW-driven variablefocus liquid microlens. The fabrication started with spin coating AZ4620 photoresist on indium-tin-oxide (ITO)-coated glass slides (\#703176, Sigma-Aldrich Corporation, St. Louis, MO). After the photoresist was photopatterned and developed, the ITO glass slide was immersed in a solution of $37 \%$ HCL, water, and $70 \% \mathrm{HNO}_{3}$ (volume ratio = 4:2:1) for $3 \mathrm{~min}$ to etch away the unprotected ITO [see Fig. 5(a)]. Following the photoresist removal, a 150-nm-thick silicon nitride (SiN) film was deposited on the electrodes by plasma-enhanced chemical vapor deposition (PECVD) [Fig. 5(b)]. Then, the SiN film above the center electrode (electrode 1) was etched away by photolithography, and the center electrode was covered by adhesive tape [see Fig. 5(c)]. Next, 1- $\mu \mathrm{m}$ hydrophobic Teflon layer (Teflon AF1600, DuPont, Wilmington, DE) was deposited on the silicon nitride by spin coating at $1000 \mathrm{r} / \mathrm{min}$ for $20 \mathrm{~s}$, and then the adhesive tape was removed [see Fig. 5(d)]. To remove the solvent in Teflon, the ITO glass slide was subject to a baking cycle as follows [32].

1) Stay at ambient temperature for 15-20 min.

2) Bake at $112^{\circ} \mathrm{C}$ for $15 \mathrm{~min}$.

3) Bake at $165^{\circ} \mathrm{C}$ for $15 \mathrm{~min}$.

4) Bake at $330{ }^{\circ} \mathrm{C}$ for $30 \mathrm{~min}$.

Finally, a cylindrical glass chamber with a 6-mm opening was fixed on the substrate by epoxy glue. An aqueous water droplet was dropped on electrode 1 and then covered by silicone oil. The cross-sectional schematic and a picture of the microlens are shown in Fig. 5(e). Due to the higher refractive index of silicone oil, this lens is diverging, and its focal length can be varied by the voltage applied on the two electrodes.

\section{EXPERIMENTAL RESULTS}

The 3-D profiling method was performed on all three types of microlenses fabricated. A complete surface profile was
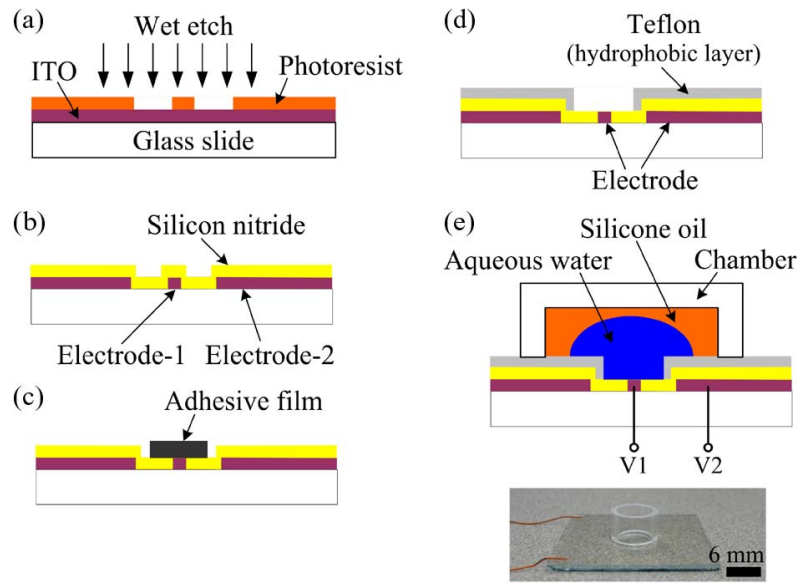

Fig. 5. Fabrication of the electrowetting-driven liquid lens. (a) An ITO-coated glass slide was used as the substrate. The thickness of the ITO film is about $20 \mathrm{~nm}$. The ITO electrode was patterned by photolithography and wet etching. (b) Silicon nitride film that was $150 \mathrm{~nm}$ thick was deposited by PECVD. (c) Silicon nitride that covers Electrode-1 was etched away. An adhesive was applied to cover Electrode-1. The thickness of the applied adhesive film is about $50 \mu \mathrm{m}$. (d) Teflon film that was $1 \mu \mathrm{m}$ was spin coated on top of the silicon nitride, and the adhesive film was removed afterward. (e) Crosssectional schematic and image of the electrowetting-based microlens. A 6-mmdiameter cylindrical glass chamber was stuck on the substrate by epoxy glue. An aqueous water droplet was dropped on Electrode-1, and the chamber was then filled with silicone oil.

obtained for the photoresist microlens. For the two types of variable-focus liquid lenses, the surface profile of each lens was measured within the central 1-mm-radius circle at different focal lengths. In addition, the multifocus measurement was dynamically achieved, which means that the optical setup needed no adjustment and the real-time surface was measured when the focal length was changing. The response time of the measurement is about $1 \sim 2 \mathrm{~s}$, limited by the response speed of the wavefront sensor.

\section{A. Photoresist Microlens Surface Profile}

The photoresist microlens was measured by the optical setup shown in Fig. 2(b). Relay lenses with $f_{1}=100 \mathrm{~mm}$ and $f_{2}=$ $600 \mathrm{~mm}$ were chosen to magnify the wavefront by a factor of 6 to fill the wavefront sensor. Fig. 6(a) is the wavefront profile reported by the built-in software of the sensor. The wavefront data were exported and then processed in MATLAB to calculate the surface profile. Fig. 6(b) illustrates the 3-D surface map of the photoresist lens. The height of the lens is $10.8 \mu \mathrm{m}$. The spherical surface [defined in (3)] fitting result is $c=0.329 \mathrm{~mm}^{-1}$ and $R=3.04 \mathrm{~mm}$, and the conical surface [defined in (4)] fitting result is $c=0.350 \mathrm{~mm}^{-1}, k=-10.09$, and $R=2.86 \mathrm{~mm}$. Because the radius of the lens aperture is small $(0.248 \mathrm{~mm})$ and the curvatures derived from two models are close, the difference between these two models is not significant. Under the spherical lens assumption, the focal length estimated by (8) is $f_{\text {spherical }}=4.67 \mathrm{~mm}$ and its relative error is $3.24 \%$ compared with the measured focal length $f=4.53 \mathrm{~mm}$. Therefore, the photoresist microlens tested is approximately a spherical lens. Spherical lenses have proven to be a good approximation for photoresist microlenses and 


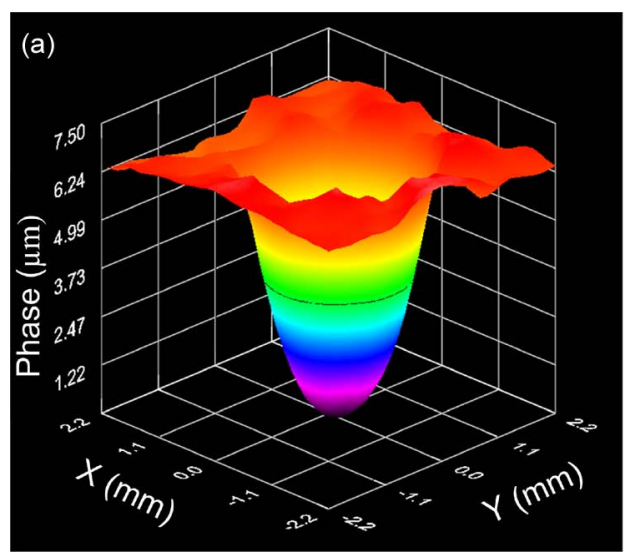

(b)

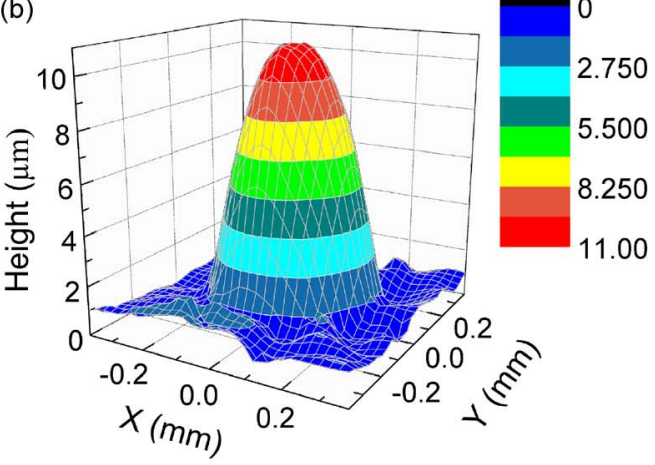

Fig. 6. (a) Wavefront map drawn directly by the built-in software of the wavefront sensor when a photoresist microlens was measured. (b) Surface profile of the photoresist microlens. The lens aperture is $496 \mu \mathrm{m}$, and its focal length is around $4.5 \mathrm{~mm}$. The height of the lens is $10.8 \mu \mathrm{m}$, and the surface profile is approximately spherical.
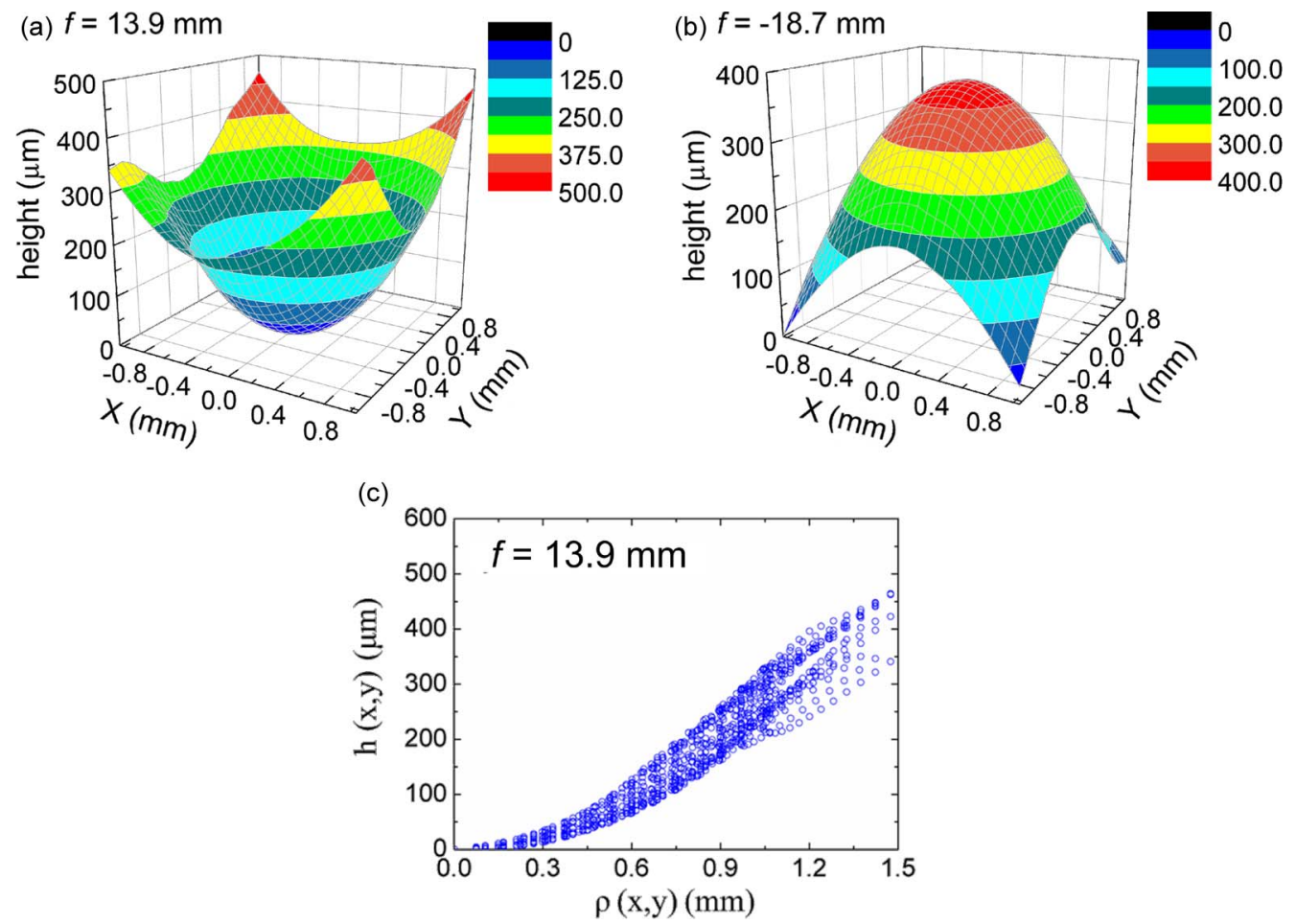

Fig. 7. (a) and (b) Surface profiles of the hydrogel-driven liquid microlens at $f=13.9 \mathrm{~mm}$ and $f=-18.7 \mathrm{~mm}$. (c) Scatter plot of surface height $h(x, y)$ at $f=13.9 \mathrm{~mm}$ versus $\rho(x, y)$, the distance from $(x, y)$ to the vertex (defined in Fig. 2). Within the area near the center, the surface profile is almost spherical, whereas at the edges of the aperture, the profile evolves to a linear pattern, and the asymmetry becomes more severe.

their shape can be well predicted by photoresist volume and fabrication parameters [33].

\section{B. Hydrogel-Driven Liquid Lens Surface Profile}

The hydrogel-driven liquid microlens was measured within a wide range of focal lengths. Relay lenses with $f_{1}=100 \mathrm{~mm}$ and $f_{2}=200 \mathrm{~mm}$ were used to magnify the wavefront by a factor of 2. First, the liquid lens functioned as a converging lens, and it was measured at $f=13.9 \mathrm{~mm}$. Then, the lens was heated by a silicone rubber heater, which was actuated by a temperature controller (CSC32 Benchtop, OMEGA Engineering Inc., Stamford, CT, USA) [15]. It was actuated to a diverging lens and was measured at $f=-18.7 \mathrm{~mm}$. The 3-D surface profiles measured at both focal lengths are shown in Fig. 7(a)-(b). As its focal length varied from $13.9 \mathrm{~mm}$ to $-18.7 \mathrm{~mm}$, the water-oil interface of the liquid lens changed from downward bending to swelling. Due to the nonuniform hydrophilicity of the aperture that resulted from the surface treatment during the fabrication, the surface profile was not completely symmetric. Fig. 7(c) illustrates the scatter plot of surface height $h(x, y)$ (defined in Fig. 2) versus the distance from point $(x, y)$ to the vertex when the lens was at $f=$ $13.9 \mathrm{~mm}$. At the center of the lens, the surface has a smooth spherical shape and a slight asymmetry. Near the edges of the aperture, the surface profile gradually evolves to a linear shape, 
TABLE I

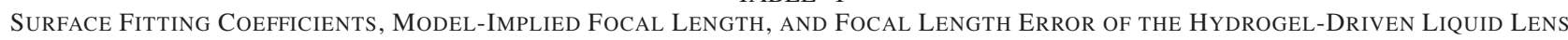

\begin{tabular}{cccccccc}
\hline & \multicolumn{3}{c}{ Spherical surface model } & \multicolumn{3}{c}{ Conical surface model } \\
\hline $\begin{array}{c}f \\
(\mathrm{~mm})\end{array}$ & $\begin{array}{c}R \\
(\mathrm{~mm})\end{array}$ & $\begin{array}{c}\text { implied } f \\
(\mathrm{~mm})\end{array}$ & $\begin{array}{c}f \text { relative } \\
\text { error }\end{array}$ & $\begin{array}{c}R \\
(\mathrm{~mm})\end{array}$ & $k$ & $\begin{array}{c}\text { implied } f \\
(\mathrm{~mm})\end{array}$ & $\begin{array}{c}f \text { relative } \\
\text { error }\end{array}$ \\
\hline 13.9 & 3.07 & 20.48 & $47.4 \%$ & 1.79 & -5.88 & 11.96 & $-13.9 \%$ \\
\hline 18.2 & 3.80 & 25.30 & $39.0 \%$ & 2.69 & -6.76 & 17.93 & $-1.5 \%$ \\
\hline-18.7 & -3.81 & -25.39 & $35.8 \%$ & -2.75 & -6.62 & -18.34 & $-1.9 \%$ \\
\hline \hline
\end{tabular}
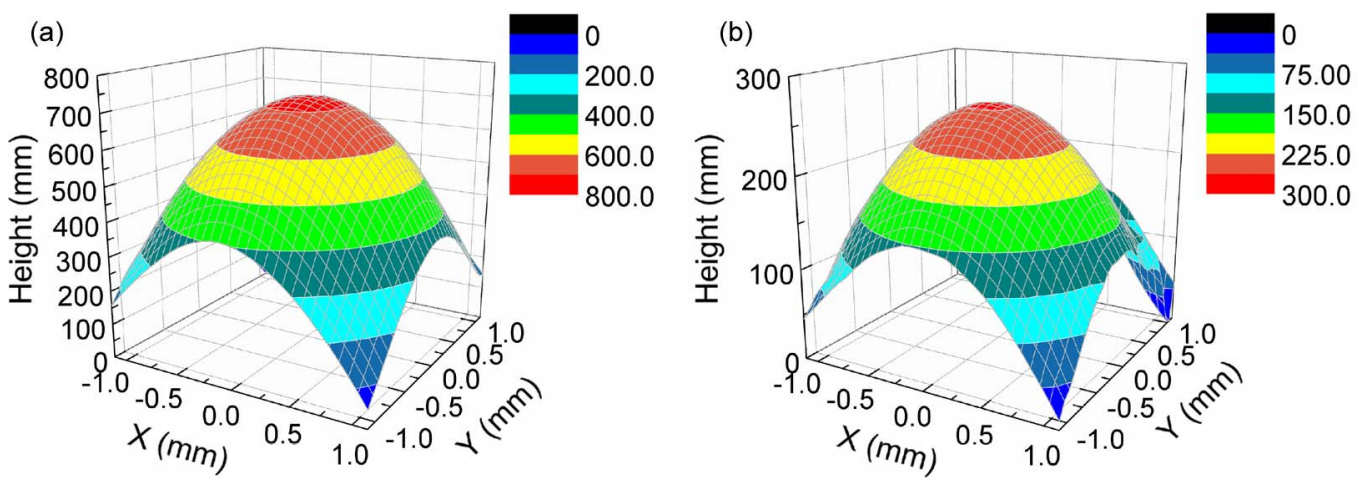

Fig. 8. Surface profiles of electrowetting-driven microlenses. (a) $f=-10.1 \mathrm{~mm}$. (b) $f=-27.5 \mathrm{~mm}$. With increasing applied voltage, the RoC of the water-oil interface became larger, and the absolute value of the focal length rose. The $\mathrm{P}-\mathrm{V}$ value of the surface profile dropped from $726 \mu \mathrm{m}$ to $266 \mu \mathrm{m}$ when $f$ was tuned from $-10.1 \mathrm{~mm}$ to $-27.5 \mathrm{~mm}$.

and the asymmetry is exacerbated. The water-oil interface is pinned at the hydrophobic-hydrophilic boundary. Closer to the aperture edges, the water surface bends further downward the hydrophilic side wall, becoming relatively more linear rather than spherical. The hydrophilicity variation results in larger asymmetry near the edges. This phenomenon was also observed at other focal lengths tested.

The surface profiles were fitted to both the spherical and the conical surface models. Table I illustrates the coefficients obtained from surface fitting and the model-implied focal length calculated by (5). The focal length implied by the spherical surface model deviated from the measured focal length by a large scale, suggesting that spherical fitting was inappropriate for this liquid lens. The negative conic constant indicates that the surface was in hyperbolic shape, matching with the observations in Fig. 7(c) that the profile becomes relatively linear at the edges.

\section{EW-Driven Liquid Lens Surface Profile}

The EW-driven microlens was also measured at different focal lengths, and the optical setup was identical to the setup for measuring the hydrogel-driven microlens. Fig. 8 shows the 3-D surface profile of the microlens when its focal length was tuned to $f=-10.1 \mathrm{~mm}$ and $f=-27.5 \mathrm{~mm}$. When the applied voltage increased, the peak-to-valley $(\mathrm{P}-\mathrm{V})$ value of the profile decreased from $726 \mu \mathrm{m}$ to $266 \mu \mathrm{m}$. The surface profiles were fitted to both spherical and conical surface models, and the results of conical surface fitting are reported in Table II.
The conic constant was significantly smaller than 0 . Therefore, the EW-driven microlens is better fitted with the conical surface model within the measured area.

\section{Estimation of Lens PERformance FROM SURFACE PROFILES}

Once the 3-D surface profiles of the microlenses are obtained, theoretically, they can be used to calculate the optical properties of the microlenses. For example, we could approximately estimate the focal length and aberrations in Zemax optical software (Zemax Development Corporation, Bellevue, WA) using the surface profiles. It is also interesting to study how accurate such estimation is. In Zemax, the lens surface can be defined by an even asphere model, which is a conical surface plus a polynomial expansion, using the even orders of radial coordinate. All the surface profiles were first fitted to the conical surface model, except that the profile of the photoresist lens was fitted to the spherical model. Then, the deviation of the conical surface from the measured surface was fitted to a polynomial equation on the order of 6 . The parameters obtained from surface fitting were subsequently used to define the lens surface in Zemax. Table III summarizes the parameters for defining the even asphere surface in Zemax, including the RoC $R$, conic constant $c$, and coefficients of polynomials.

Fig. 9 shows the Zemax-simulated modulation transfer function (MTF) of each type of microlens. The MTF describes the overall lens performance. A lens generally has better optical performance when its MTF is closer to the MTF of 
TABLE II

Conical Surface Fitting Coefficients, Implied Focal Length, And Focal Length Error of the EW-Driven LiQuid Lens

\begin{tabular}{ccccc}
\hline \hline$f(\mathrm{~mm})$ & $R(\mathrm{~mm})$ & $k$ & $\operatorname{implied} f(\mathrm{~mm})$ & $f$ relative error \\
\hline-10.1 & 1.66 & -1.61 & -11.09 & $9.8 \%$ \\
\hline-27.5 & 4.33 & -6.31 & -28.84 & $4.9 \%$ \\
\hline \hline
\end{tabular}

TABLE III

Parameters of Even Asphere Surface Defined in Zemax

\begin{tabular}{lcccccc}
\hline \multicolumn{2}{c}{ Lens types } & \multicolumn{5}{c}{ Even asphere model } \\
\hline & $f(\mathrm{~mm})$ & $R(\mathrm{~mm})$ & $k$ & $r^{6}$ & $r^{4}$ & $r^{2}$ \\
\hline $\begin{array}{c}\text { Photoresist } \\
\text { lens }\end{array}$ & 4.5 & 3.04 & 0.00 & -29.067 & 1.739 & 0.007 \\
\hline $\begin{array}{c}\text { Hydrogel- } \\
\text { driven } \\
\text { lens }\end{array}$ & 13.9 & 1.79 & -5.88 & -0.013 & 0.040 & -0.029 \\
\hline $\begin{array}{c}\text { Electrowetting } \\
\text { lens }\end{array}$ & -10.1 & -1.69 & -6.76 & -0.011 & 0.038 & -0.036 \\
\hline \hline
\end{tabular}

a diffraction-limit lens. For the hydrogel-driven lens at $f=$ $18.2 \mathrm{~mm}$ [Fig. 9(c)] and the EW-driven lens at $f=-28.7 \mathrm{~mm}$ [Fig. 9(f)], the MTF of the microlens almost overlaps the diffraction limit, indicating that the wavefront aberration is very small. The wavefront aberration increases with increasing surface curvature; therefore, the MTF deviates from its diffraction limit, as observed in other cases in Fig. 9.

Table IV summarizes the Zemax-simulated rms wavefront error and the focal length of each type of microlens. The $f$-number $(f / \#)$ in the table is given by

$$
f / \#=\frac{f}{D}
$$

where $f$ is the focal length, and $D$ is the diameter of the entrance pupil of a microlens. The Zemax-simulated focal length is very close to the experimental result for photoresist microlens. For the two variable-focus liquid microlenses, the error of focal length becomes relatively smaller as $f$ /\# increases. The wavefront aberration is dominated by spherical aberrations, and it decreases with increasing $f / \#$. At higher $f / \#$, the microlens has smaller surface bending (larger RoC) and, therefore, less spherical aberration [34]. The primary spherical aberration is described by the Zernike coefficient $c_{40}$ in the Zernike standard polynomials [35]. The Zernike coefficients are reported by both the Shack-Hartmann wavefront sensor and the Zemax simulation. For hydrogel-driven liquid lens, $c_{40}$ is reported in Table V. The simulation results are not very close to the experimental results, and the relative error can be larger than $10 \%$.

Based on the comparisons shown in Tables IV and V between the Zemax estimation and the experimental results from the wavefront sensor, we conclude that the Zemax simulation provides only a rough estimate of the optical properties of the microlenses. The wavefront sensor can directly measure the aberrations of a microlens. However, for the Zemax simulation based on the fitted surface profile, the error could be from two sources. One error source is the wavefront measurement. In principle, the wavefront right behind the liquid-liquid interface should be measured to derive the surface profile. However, a transparent thin substrate is needed to package the lens, and the wavefront behind the substrate was measured and used for surface profile calculation. Another error source, which could potentially be significant, is the surface fitting process, which is inherently an approximation. The measured surface profile was fitted to a conical model, which is rotation symmetric; however, the real surface could be more complicated and could suffer rotational nonsymmetry. Further investigation is needed to improve the accuracy of the measurement and to study other surface fitting models.

\section{Discussion of the Optical Setup}

Note that the optical setup described in Section III is restricted by certain limits. First, the optical setup in Fig. 3(a) is for obtaining rough surface profiles of microlenses. In the reference measurement, a collimated beam reaches the wavefront sensor, whereas in the microlens characterization measurement, the beam that emerges from $\mathrm{L}_{2}$ is converging. The path difference could decrease the accuracy of the wavefront measurement. When aberration measurements of high accuracy are required, conventional optical setup using the Shack-Hartmann wavefront is recommended [29], [35].

Second, relay lenses with nonunity magnification are used in the optical setup. Therefore, care must be taken in the alignment to avoid any curvature added by the relay lenses. The effect of changing the illumination area on the relay lenses can add deviations from flatness. The surface irregularity of the lens is approximately a quarter of a wavelength, which is in the submicrometer range, assuming $\lambda=593 \mathrm{~nm}$. The deviations are relatively small, however, compared to the overall surface profiles measured.

In addition, to obtain consistent result from the wavefront sensor, the wavefront that emerges from the microlens under test cannot exceed the dynamic range of the wavefront sensor. The dynamic range of the wavefront sensor in Fig. 3(a) is $100 \times$ $\lambda$, where $\lambda$ is the wavelength. The phase due to a simple lens is given by [26]

$$
\phi(x, y)=\frac{-k}{2 f}\left(x^{2}+y^{2}\right)
$$




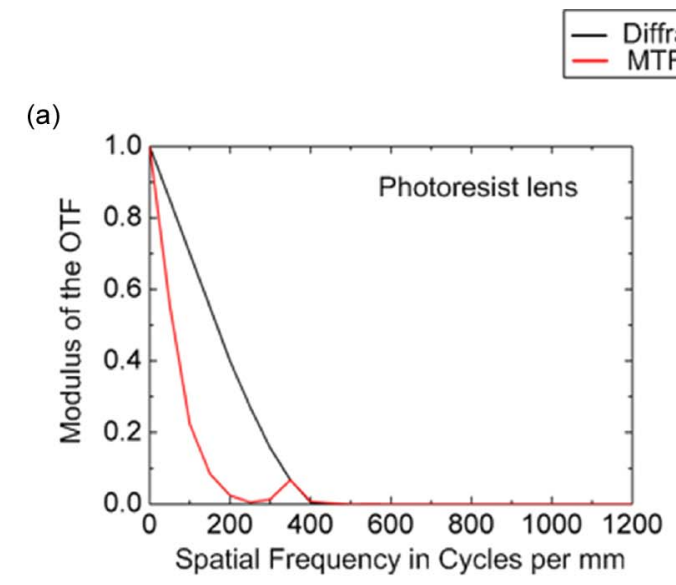

(b)

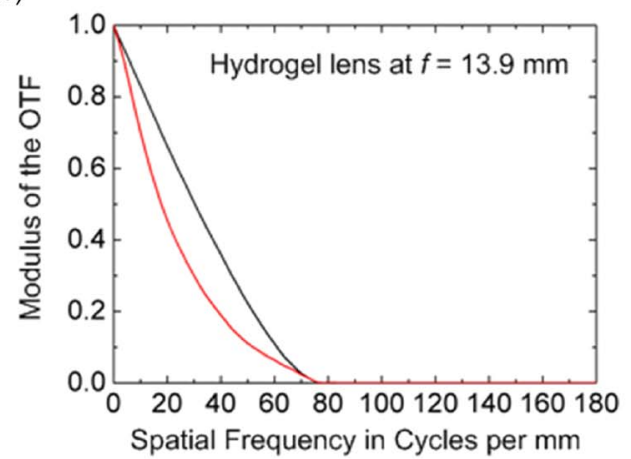

(c)

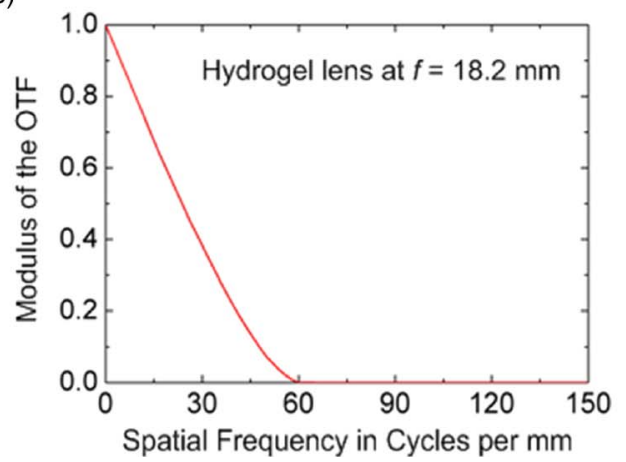

(d)

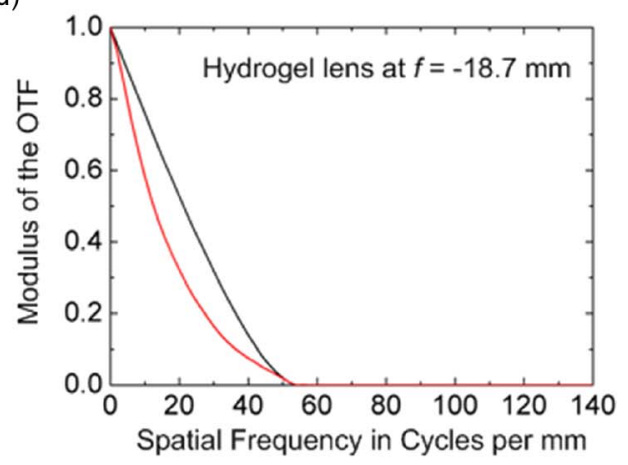

(e)

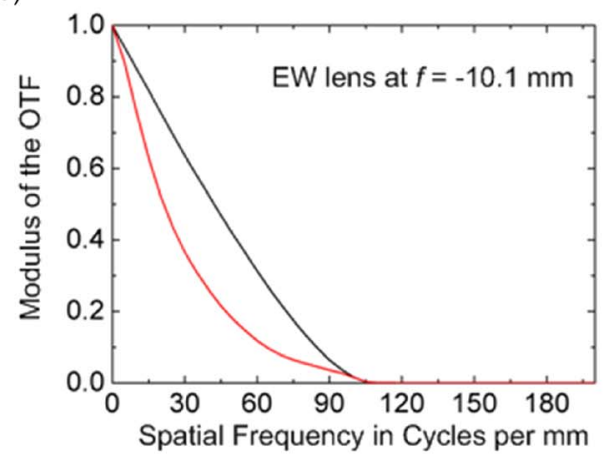

(f)

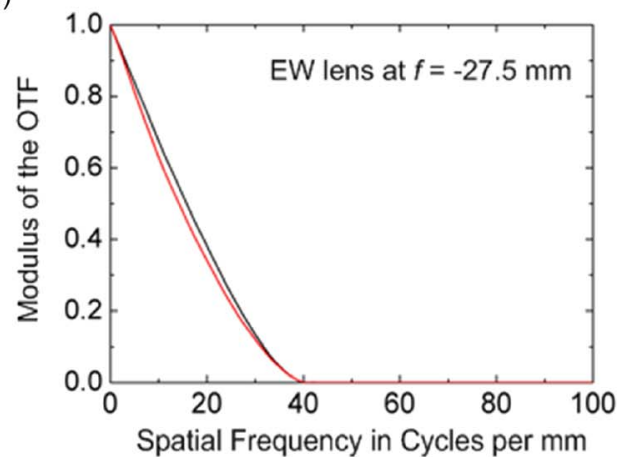

Fig. 9. MTF of the microlenses. (a) MTF of the photoresist microlens. (b)-(d) MTF of the hydrogel-driven microlens at $f=13.9 \mathrm{~mm}, f=18.2 \mathrm{~mm}$, and $f=-18.7 \mathrm{~mm}$. (e) and (f) MTF of the EW microlens at $f=-10.1 \mathrm{~mm}$ and $f=-27.5 \mathrm{~mm}$. In (c), the MTF of the hydrogel-driven liquid lens and its diffraction limit almost overlap at $f=18.2 \mathrm{~mm}$, and the two profiles cannot be differentiated in the figure.

The beam size is $2 \mathrm{~mm}$; therefore, the maximum of $\left(x^{2}+y^{2}\right)$ is $1 \mathrm{~mm}^{2}$. For $f=10 \mathrm{~mm}$ and $\lambda=593 \mathrm{~nm}$, the maximum phase is therefore $\phi_{\max }=-168.6 \pi$, corresponding to a wavefront of $-84.3 \lambda$, which is well within the dynamic range of the wavefront sensor. When a microlens with high optical power will be tested, it is important to check that the wavefront that emerges from the microlens is within the dynamic range of the wavefront sensor.

Finally, the major importance of the principles in Section II and the optical setup in Section III is to dynamically measure the rough shape of a variable-focus microlens without changing optics. This method visualizes what is going on with the liquid-liquid interface and dynamically measures it as the focal length of the lens is adjusted, which is an advantage over methods that require changing optics and realigning in between.

\section{CONCLUSION}

We have demonstrated a 3-D surface profiling method using the Shack-Hartmann wavefront sensor that can be applied to both solid and liquid microlenses. The surface profile is calculated from the wavefront measured by the wavefront sensor. The principles and optical setup are presented and verified by the test of a commercial spherical lens. The resolution of this profiling method is determined by the aperture of the lenslet in the wavefront sensor, and the relay lenses in the setup needs to be well chosen to match the aperture of the microlens with the size of the CCD in the wavefront sensor. Three types of microlenses were fabricated and measured: one solid fixedfocus photoresist microlens and two variable-focus liquid microlenses that are driven by thermoresponsive hydrogel and $\mathrm{EW}$, respectively. The two variable-focus lenses were measured 
TABLE IV

$f$-NUMBER, ZEMAX-SiMUlATED RMS WAVEFront ERROR, AND FOCAL LENGTH

\begin{tabular}{ccccc}
\hline & Lens types & & \multicolumn{2}{c}{$\begin{array}{c}\text { Zemax-simulated } \\
\text { lens performance }\end{array}$} \\
\hline & $\begin{array}{c}f \\
(\mathrm{~mm})\end{array}$ & $f$ l\# & $\begin{array}{c}\text { RMS } \\
\text { error } \\
\text { (wave) }\end{array}$ & $\begin{array}{c}f \\
(\mathrm{~mm})\end{array}$ \\
\hline $\begin{array}{c}\text { Photoresist } \\
\text { lens }\end{array}$ & 4.5 & 11.3 & 0.191 & 4.48 \\
\hline $\begin{array}{c}\text { Hydrogel- } \\
\text { driven } \\
\text { lens }\end{array}$ & 18.2 & 9.1 & 0.002 & 18.66 \\
\hline $\begin{array}{c}\text { Electrowetting } \\
\text { lens }\end{array}$ & -18.7 & 9.4 & 0.017 & -19.12 \\
\hline
\end{tabular}

TABLE V

ZERNiKe COEFFICIENT $c_{40}$ OF THE HYDROGEL-DRIVEN MiCROLENS REPORTED BY ZEMAX AND THE WAVEFRONT SENSOR

\begin{tabular}{ccc}
\hline \hline$f$ & $\mathrm{c}_{40}$ & $\mathrm{c}_{40}$ \\
$(\mathrm{~mm})$ & reported by Zemax (wave) & reported by wavefront sensor (wave) \\
\hline 13.9 & -1.49 & -1.38 \\
\hline 18.2 & -0.745 & -0.541 \\
\hline-18.7 & 0.568 & 0.715 \\
\hline \hline
\end{tabular}

at different focal lengths. The obtained surface profiles were fitted to spherical and conical surface models, and the modelimplied focal lengths were compared with experiment results. The surface fitting results were also used in optical analysis software to simulate the optical properties of the microlenses; however, the comparison between the simulation and the experimental results showed that the simulation gave only a rough estimate of the optical properties of the microlenses due to the errors generated by the wavefront approximation and the surface fitting procedures. The 3-D surface profiling method presented should help in obtaining a deeper understanding of the surface deformation in a variable-focus liquid microlens and guiding microlens design and fabrication.

In the future, we will further develop this surface profiling technique and apply the measured surface profiles to improve the design and fabrication of hydrogel- and EW-driven liquid microlenses, e.g., the surface wettability, aberration reduction, and uniformity across lenses in a microlens array. In addition, this method could potentially be applied to the characterization of general liquid-liquid interfaces in microfluidics. However, note that the wavefront measurement is limited by the dynamic range and local RoC (gradient) limit of the Shack-Hartmann wavefront sensor.

\section{ACKNOWLEDGMENT}

The authors would like to thank Prof. A. Sheinis, B. Aldalali, J. Hartmann, and M. Meng for the discussions and their assistance. This research utilized NSF-supported shared facilities at the University of Wisconsin, Madison.

\section{REFERENCES}

[1] S. Kuiper and B. H. W. Hendriks, "Variable-focus liquid lens for miniature cameras," Appl. Phys. Lett., vol. 85, no. 7, pp. 1128-1130, Aug. 2004.

[2] B. Berge and J. Peseux, "Variable-focal lens controlled by an external voltage: An application of electrowetting," Eur. Phys. J. E, Soft Matter, vol. 3, no. 2, pp. 159-163, Oct. 2000.

[3] J. J. Snyder, P. Reichert, and T. M. Baer, "Fast diffraction-limited cylindrical microlenses," Appl. Opt., vol. 30, no. 19, pp. 2743-2747, Jul. 1991.

[4] P. Savander and H.-J. Haumann, "Microlens array used for collimation of linear laser-diode array," Meas. Sci. Technol., vol. 4, no. 4, pp. 541-543, Apr. 1993.

[5] M. C. Hutley, P. Savander, and M. Schrader, "The use of microlenses for making spatially variant optical interconnections," Pure Appl. Opt., vol. 1, no. 6, pp. 337-346, Nov. 1992.

[6] J. Jahns and S. J. Walker, "Two-dimensional array of diffractive microlenses fabricated by thin-film deposition," Appl. Opt., vol. 29, no. 7, pp. 931-936, Mar. 1990.

[7] M. H. Wu and G. M. Whitesides, "Fabrication of arrays of twodimensional micropatterns using microspheres as lenses for projection photolithography," Appl. Phys. Lett., vol. 78, no. 16, pp. 2273-2275, Apr. 2001.

[8] R. Völkel, H. P. Herzig, P. Nussbaum, R. Dandliker, and W. B. Hugle, "Microlens array imaging system for photolithography," Opt. Eng., vol. 35, no. 11, pp. 3323-3330, Nov. 1996.

[9] H. Choo and R. S. Muller, "Devices, structures, and processes for optical MEMS," IEEJ Trans. Elect. Electron. Eng., vol. 2, no. 3, pp. 216-231, May 2007.

[10] P. Nussbaum, R. Völkel, H. P. Herzig, M. Eisner, and S. Haselbeck, "Design, fabrication and testing of microlens arrays for sensors and microsystems," Pure Appl. Opt., vol. 6, no. 6, pp. 617-636, Nov. 1997.

[11] Z. D. Popovic, R. A. Sprague, and G. A. Connell, "Technique for monolithic fabrication of microlens arrays," Appl. Opt., vol. 27, no. 7, pp. 12811284, Apr. 1988.

[12] S. Yang, T. N. Krupenkin, P. Mach, and E. A. Chandross, "Tunable and latchable liquid microlens with photopolymerizable components," Adv. Mater, vol. 15, no. 11, pp. 940-943, Jun. 2003.

[13] F. Krogmann, W. Monch, and H. Zappe, "A MEMS-based variable microlens system," J. Opt. A, Pure Appl. Opt., vol. 8, no. 7, pp. S330-S336, Jul. 2006.

[14] L. Dong, A. K. Agarwal, D. J. Beebe, and H. Jiang, "Adaptive liquid microlenses activated by stimuli-responsive hydrogels," Nature, vol. 442 , no. 7102 , pp. 551-554, Aug. 2006.

[15] X. Zeng, C. Li, D. Zhu, H. J. Cho, and H. Jiang, "Tunable microlens arrays actuated by various thermoresponsive hydrogel structures," J. Micromech. Microeng., vol. 20, no. 11, pp. 115 035-115045, Nov. 2010.

[16] D. Zhu, C. Li, X. Zeng, and H. Jiang, "Tunable-focus microlens arrays on curved surfaces," Appl. Phys. Lett., vol. 96, no. 8, pp. 081111-1-081111-3, Feb. 2010.

[17] N. Chronis, G. L. Liu, K. H. Jeong, and L. P. Lee, "Tunable liquid-filled microlens array integrated with microfluidic network," Opt. Exp., vol. 11, no. 19 , pp. 2370-2378, Sep. 2003.

[18] A. Werber and H. Zappe, "Tunable microfluidic microlenses," Appl. Opt., vol. 44, no. 16, pp. 3238-3245, Jun. 2005.

[19] H. Ren and S. T. Wu, "Variable-focus liquid lens," Opt. Exp., vol. 15, no. 10, pp. 5931-5936, May 2007.

[20] G. Beadie, M. L. Sandrock, M. J. Wiggins, R. S. Lepkowicz, J. S. Shirk, M. Y. Ponting, Y. Yang, T. Kazmierczak, A. Hiltner, and E. Baer, "Tunable polymer lens," Opt. Exp., vol. 16, no. 16, pp. 11 847-11 857, Jul. 2008.

[21] S. Xu, Y. Liu, H. Ren, and S. Wu, "A novel adaptive mechanical-wetting lens for visible and near-infrared imaging," Opt. Exp., vol. 18, no. 12, pp. 12430-12 435, Jun. 2010.

[22] X. Zeng and H. Jiang, "Polydimethylsiloxane microlens arrays fabricated through liquid-phase photopolymerization and molding," J. Microelectromech. Syst., vol. 17, no. 5, pp. 1210-1217, Oct. 2008.

[23] C. B. Gorman, H. A. Biebuyck, and G. M. Whitesides, "Control of the shape of liquid lenses on a modified gold surface using an applied electrical potential across a self-assembled monolayer," Langmuir, vol. 11, no. 6, pp. 2242-2246, Jun. 1995. 
[24] P. M. Moran, S. Dharmatilleke, A. H. Khaw, K. W. Tan, M. L. Chan, and I. Rodriguez, "Fluidic lenses with variable focal length," Appl. Phys. Lett., vol. 88, no. 4, pp. 041120-1-041120-3, Jan. 2006.

[25] C. Li, G. Hall, X. Zeng, D. Zhu, K. Eliceiri, and H. Jiang, "Threedimensional surface profiling and optical characterization of liquid microlens using a Shack-Hartmann wavefront sensor," Appl. Phys. Lett., vol. 98, no. 17, pp. 171 104-1-171 104-3, Apr. 2011.

[26] J. W. Goodman, Introduction to Fourier Optics, 3rd ed. Greenwood Village, CO: Roberts \& Co., 2005.

[27] T. M. Jeong, M. Menon, and G. Yoon, "Measurement of wavefront aberration in soft contact lenses by use of a Shack-Hartmann wavefront sensor," Appl. Opt., vol. 44, no. 21, pp. 4523-4527, Jul. 2005.

[28] P. D. Pulaski, J. P. Roller, D. R. Neal, and K. Ratte, "Measurement of aberrations in microlenses using a Shack-Hartmann wavefront sensor," in Curr. Dev. Lens Des. Opt. Eng. III-Proc. SPIE, R. Fischer, W. Smith, and B. Johnson, Eds., 2002, vol. 4767, pp. 44-52.

[29] C. Du, K. Zuerl, and J. Schwider, "Wavefront characterization with a miniaturized Shack-Hartmann sensor," Optik, vol. 101, no. 4, pp. 151156, Feb. 1996.

[30] J. Pfund, N. Lindlein, J. Schwider, R. Burow, T. Blümel, and K. E. Elssner, "Absolute sphericity measurement: A comparative study of the use of interferometry and a Shack-Hartmann sensor," Opt. Lett., vol. 23, no. 10, pp. 742-744, May 1998.

[31] D. R. Neal, J. Copland, and D. A. Neal, "Shack-Hartmann wavefront sensor precision and accuracy," in Adv. Characterization Tech. Opt. Semicond. Data Storage Compon.-Proc. SPIE, A. Duparré and B. Singh, Eds., 2002, vol. 4779, pp. 148-160.

[32] A. Datta, I. Y. Eom, A. Dhar, P. Kuban, R. Manor, I. Ahmad, S. Gangopadhyay, T. Dallas, M. Holtz, and H. Temkin, "Microfabrication and characterization of Teflon AF-coated liquid core waveguide channels in silicon," IEEE Sensors J., vol. 3, no. 6, pp. 788-795, Dec. 2003.

[33] D. Daly, Microlens Arrays. Boca Raton, FL: CRC Press, 2000.

[34] R. E. Fischer, B. Tadic-Galeb, and P. R. Yoder, Optical System Design, 2nd ed. New York: McGraw-Hill, 2008.

[35] D. Malacara, Optical Shop Testing. New York: Wiley-Blackwell, 2007.

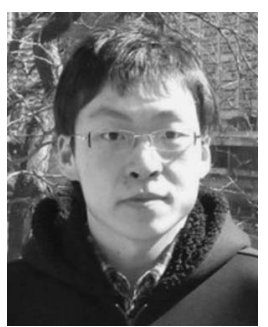

Chenhui Li received the B.S. degree in electrical engineering in 2007 from Tsinghua University, Beijing, China, and the M.S. degree in electrical and computer engineering in 2010 from the University of Wisconsin, Madison, where he is currently working toward the $\mathrm{Ph} . \mathrm{D}$. degree in electrical engineering in the Department of Electrical and Computer Engineering.

His research interests include microoptical imaging, liquid focus-variable microlenses, electrowetting, and light-field cameras.

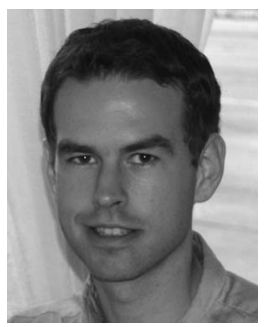

Gunnsteinn Hall received the B.S. degree in electrical engineering and physics in 2007 from the University of Iceland, Reykjavík, Iceland, and the M.S. degree in biomedical engineering in 2010 from the University of Wisconsin, Madison, where he is currently working toward the Ph.D. degree in biomedical engineering in the Department of Biomedical Engineering and the Laboratory for Optical and Computational Instrumentation.

His research interests include adaptive optics, wavefront sensing, and applications of nonlinear

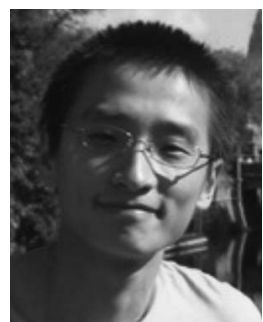

Difeng Zhu received the B.S. degree in microelectronics from Peking University, Beijing, China, in 2007, and the Ph.D. degree in electrical engineering from the University of Wisconsin, Madison, in 2011.

$\mathrm{He}$ is currently an R\&D Process Engineer with Micron Technology Inc., Boise, ID. His research interests include liquid microlens array, microelectronics, and MEMS fabrication.

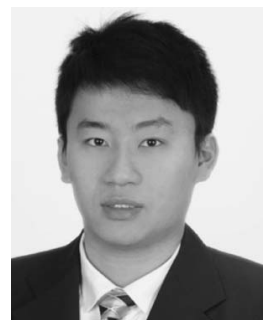

Heng $\mathbf{~} \mathbf{i}$ is currently working toward the B.S. degree in electrical engineering and applied mathematics in the Department of Electrical and Computer Engineering, University of Wisconsin, Madison, WI.

$\mathrm{He}$ is also an Undergraduate Researcher with the Micro-Nano Sensors and Actuators Laboratory, Department of Electrical and Computer Engineering, University of Wisconsin.

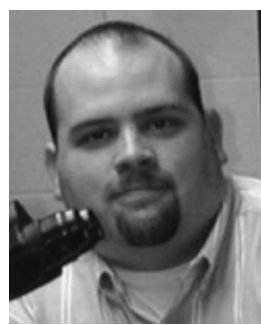

Kevin W. Eliceiri received the B.S. degree in bacteriology and the M.S. degree in microbiology and biotechnology from the University of Wisconsin, Madison, where he worked on developing imaging approaches for the model nematode C. elegans under the supervision of Prof. John White, and further postgraduate training in computer science and $\mathrm{mi}-$ croscopy from the National Integrated Microscopy Resource, Madison.

Since 2000, he has been with the Laboratory for Optical and Computational Instrumentation (LOCI), University of Wisconsin, where he is currently the Director, and he is also a Principal Investigator with the Laboratory of Cell and Molecular Biology and the Department of Biomedical Engineering. His research interests include the development of novel optical imaging methods for investigating cell signaling and cancer progression, and the development of software for multidimensional image analysis.

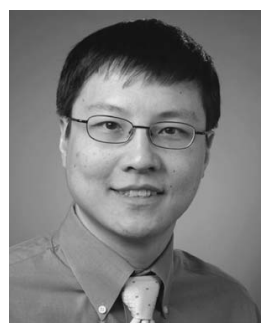

Hongrui Jiang (S'98-M'02-SM'10) received the B.S. degree in physics from Peking University, Beijing, China, and the M.S. and Ph.D. degrees in electrical engineering from Cornell University, Ithaca, NY, in 1999 and 2001, respectively.

From 2001 to 2002, he was a Postdoctoral Researcher with the Berkeley Sensor and Actuator Center, University of California, Berkeley. He is currently an Associate Professor in the Department of Electrical and Computer Engineering, a Faculty Affiliate with the Department of Biomedical Engineering, a Faculty Member of the Materials Science Program, and a Member of the Eye Research Institute, University of Wisconsin, Madison. He is currently a Member of the editorial board of the Journal of MicROELECTROMECHANICAL SYSTEMS. His research interests include microfabrication technology, biological and chemical microsensors, microactuators, optical microelectromechanical systems, smart materials and microstructures/nanostructures, labon-a-chip, and biomimetics and bioinspiration.

Dr. Jiang was the recipient of a National Science Foundation Faculty Early Career Development (CAREER) Award and the Defense Advanced Research Project Agency Young Faculty Award in 2008, the H. I. Romnes Faculty Fellowship from the University of Wisconsin, Madison, in 2011, and the National Institutes of Health (NIH) Director's New Innovator Award in 2011. 